\title{
Enhancing Urban Mobility: Integrating Ride-sharing and Public Transit
}

\author{
Mitja Stiglic Niels Agatz Martin Savelsbergh $\quad$ Mirko Gradisar
}

July 5, 2016

\begin{abstract}
Seamless integration of ride-sharing and public transit may offer fast, reliable, and affordable transfer to and from transit stations in suburban areas thereby enhancing mobility of residents. We investigate the potential benefits of such a system, as well as the ride-matching technology required to support it, by means of an extensive computational study.
\end{abstract}

\section{Introduction}

People all around the world use private cars to travel to work. Most of these commuter trips are singleoccupant vehicle trips. In the U.S., for example, single-occupant trips represent approximately $77 \%$ of all commuter trips [Polzin and Pisarski, 2013]; similar percentages are found in Europe [European Environment Agency, 2010]. The low vehicle occupancy rates combined with the high number of trips during peak-hours often leads to severe traffic congestion in urban areas. The resulting stress and air pollution, caused by vehicle emissions, can have serious negative health effects.

To reduce the negative externalities of car travel, local governments encourage the use of public transit. Unfortunately, many suburban and rural areas are not adequately served as they lack the population density to justify public transit, i.e., the public transit is not economically viable. In cities with sprawling suburban areas, the utilization of public transit to commute to work is often low, e.g., less than $5 \%$ in metropolitan areas like Houston and Atlanta [McKenzie, 2010].

To attract more riders from suburban areas to public transit, transportation agencies must find adequate solutions to accommodate the first and last mile from the riders' home to and from the transit stations. Possible solutions for a transportation agency include operating a fleet of demand-responsive feeder vehicles and collaborating with local taxi service providers. In the U.S., for example, public transport providers have 
started collaborating with Uber and Lyft to better coordinate their service offering [Murphy, 2016]. While the services provided by Uber and Lyft are convenient for the riders, they are often (too) costly for the transportation agency and/or the riders.

A cheaper and more environmentally sustainable alternative is to use already existing trips as a feeder for public transit. A recent TRB report highlights ride-sharing as an important opportunity for transportation agencies to address the "last mile problem" [Murray et al., 2012]. Deutsche Bahn (German railways) is running several pilots to synchronize bus and ride-shares to provide convenient door-to-door transportation to their travelers (Annual report DB 2015). The Flinc ride-sharing smartphone app will soon integrate train timetables and regional public transport schedules across Germany.

In this paper, we examine the potential benefits of integrating ride-sharing and public transit. Ridesharing and public transit can, in fact, complement each other. On the one hand, ride-sharing can serve as a feeder system that connects less densely populated areas to public transit. One the other hand, the public transit system can extend the reach of ride-sharing and reduce the detours of the drivers. As such, it may help to overcome incompatibilities in the itineraries of drivers and riders and facilitate the matching process.

Consider the example depicted in Figure 1 with driver $d_{1}$ and rider $r_{1}$ and two stations $s_{1}$ and $s_{2}$, where circles indicate origins and squares indicate destinations, where the number above an arc represents the time it takes to travel between the nodes, and where the driver is willing to accept an increase in trip time of at most five minutes. Without the use of public transit, a match between $d_{1}$ and $r_{1}$ is not feasible because the

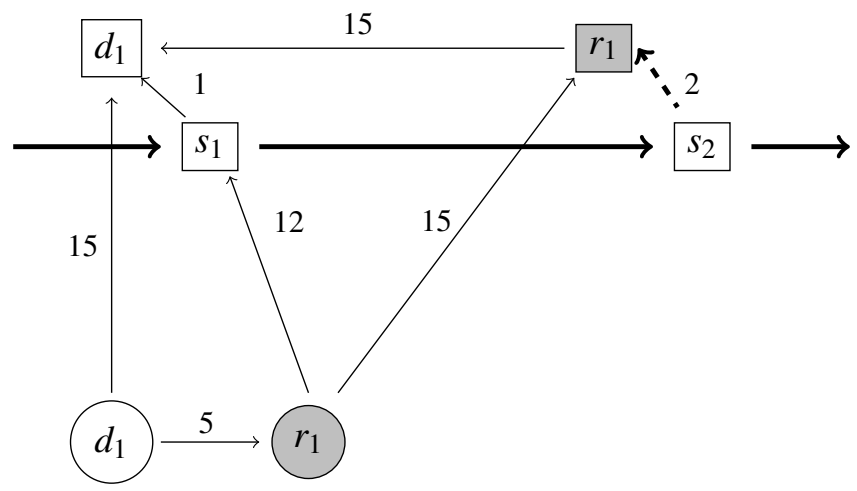

Figure 1: Rider (grey) and Driver (white) traveling from Origin (circle) to Destination (square) via Public Transit

required increase in trip time ( $20 \mathrm{~min}$ ) exceeds the driver's limit. If, however, the rider is willing to take the transit line from $s_{1}$ to $s_{2}$ and walk 2 minutes to his final destination, a feasible match between $d_{1}$ and $r_{1}$ is possible, because $d_{1}$ has to make a smaller detour. (We have to make sure that the rider can reach his final destination in time based on the timetable of the transit line.)

We consider a centralized system that automatically establishes matches between drivers and riders. A 
driver can either move a rider from his origin directly to his destination or to a transit station so that he can take the train to his final destination. The transit system operates a fixed time table and drivers and riders announce their itineraries and time schedules on short notice. In this paper, we assume the system creates matches in a way that maximizes the number of matched riders. As a secondary objective, the system minimizes the additional driving distance of the matched drivers. As such, this hierarchy aims to maximize rider mobility while minimizing the inconvenience of the matched drivers and, at the same time, the negative externalities of car travel.

When there is a park and ride facility at the station, a driver may even decide to park his car after taking a rider to the station and take public transit to his final destination himself. Drivers provide the ride in exchange for a small fee that covers the costs of operating the vehicle and a small remuneration for the time that has been "lost" (due to pick-up and drop-off as well as the detour). The public transit provider may choose to cooperate with other stakeholders and offer additional benefits to drivers that are willing to accommodate riders. These might come in the form of a free park-and-ride ticket, a toll waiver, an HOV lane permit, or priority parking in the city center. As such, this service can be offered at a relatively low cost to the rider. This may help to increase rider-ship on public transit (which will lead to an increase in public transportation revenues) and decrease the number of personal vehicles on the road.

The main contributions of our research are that (i) we introduce a new and relevant problem that considers the integration and synchronization of ride-sharing and scheduled public transit, (ii) we present a solution approach to optimally create single or multi-modal ride-share matches, and (iii) we conduct an extensive numerical study on artificial instances that capture the main characteristics of many real-world transit settings and quantify the benefits of integrating ride-sharing and public transit.

The remainder of the paper is organized as follows. In the next section, we discuss relevant and related literature. In Sections 3 and 4, we formally define the problem and introduce a solution approach. In Section 5 , we discuss the details of our numerical experiments and present the main results. We close the paper with a summary of our findings and recommendations for practitioners.

\section{Literature}

The use of demand-responsive transportation services to enhance the performance of public transportation systems has been promoted for some time and a wide variety of such services is offered in practice, often focusing on, or reserved for, people with disabilities and subsidized by the government. 
Cayford and Yim [2004] present a basic demand responsive feeder system for the city of Milbrae, California. In low-demand periods, buses can deviate from their "fixed" routes as well as from their scheduled departure times based on actual demand. The route deviation is limited to skipping portions of the fixed route when there is no demand for drop-offs on those portions on a particular trip. Buses operate according to the fixed schedule in high-demand periods.

One of the common integration options between a fixed-schedule system and an on-demand feeder system is the so-called Demand Responsive Connector (DRC). Koffman [2004] presents examples of DRCs in several US cities and observes that it is one of the most popular types of flexible transit services. Such systems typically operate within a service area and move passengers to and from a transfer point that connects to a major fixed-route transit network.

A critical question in this context is under what conditions is it better, in terms of service and cost, to operate the feeder system using a fixed route policy and under what conditions is it better to operate the feeder system as a demand-responsive service. Quadrifoglio and Xiugang [2009] and Li and Quadrifoglio [2010] develop analytical and simulation models for this purpose and find that the switching point between a demand responsive and fixed route policy is in the range from 10 to 50 customers $/ \mathrm{mi}^{2} / \mathrm{h}$.

A DRC is typically deployed within a specified zone, where the zone has one transfer point and where one transportation company provides the service. Li and Quadrifoglio [2011] consider the optimal zone design problem and develop an analytical model based on continuous approximations. Lee and Savelsbergh [2014] study a setting in which a zone has multiple transfer stations and a passenger can be dropped of at any of these transfer stations as long as the passenger's desired service is met. They demonstrate that a more flexible system can offer substantial cost savings, especially when transit services are frequent and/or transit stations are relatively close together.

In this paper we consider the use of ride-sharing as a feeder system for scheduled transit. Several recent papers focus on algorithmic approaches to optimally match riders and drivers in the ride-sharing context. The work in this area typically considers door-to-door trips in which a driver moves one or more riders from their origin to their destination (see for example Agatz et al. [2011], Wang et al. [2015], Lee and Savelsbergh [2015], and Stiglic et al. [2016]). Furuhata et al. [2013] and Agatz et al. [2012] provide a detailed overview of this line of research and the links to other modes of transportation.

In Stiglic et al. [2015], we do not require door-to-door transportation but allow riders to walk to and from meeting points to facilitate more convenient rides for the drivers. Here, we extend this work by also allowing transfers to a transit service with a fixed schedule. While there are only a few meeting points within walking distance of a specific rider, many more transit stations are within reach by using scheduled 
transit. The synchronization of the ride-share trips with the transit service links our work to multi-hop ridesharing which involves the temporal synchronization of different ride-share trips (see Herbawi and Weber [2011], Coltin and Veloso [2013] and Drews and Luxen [2013] for algorithmic approaches to these types of problems).

\section{Problem definition}

We consider a transit service provider that receives a sequence of trip announcements $A$ from participants. The set of announcements $A$ can be partitioned into the set of trip announcements by the drivers, $D \subset A$, and the set of trip announcements by the riders, $R \subset A$. A trip announcement $a \in A$ has an origin location, $o_{a}$, and a destination location, $d_{a}$, as well as an earliest departure time, $e_{a}$, at the original location and a latest arrival time, $l_{a}$, at the destination location. We assume that the departure times of the participants are somewhat flexible so that the difference $l_{a}-e_{a}$ is greater than the travel time from the origin to the destination. For each $a \in A$, there is also a maximum acceptable trip duration, $T_{a}$, a maximum acceptable walking distance from a transit station, $m_{a}$, and a maximum acceptable waiting time at a transit station, $W_{a}$. We denote the distance from location $i$ to $j$ by $d_{i j}$, the vehicle travel time from location $i$ to $j$ by $t_{i j}$, and the time it takes to walk from $i$ to $j$ by $\hat{t}_{i j}$. We assume that a time $\tau$ is incurred when a driver picks up a rider.

We consider a connected public transit network with a fixed cyclic timetable. The timetable describes the departure and arrival times of each train at each station. (We refer to the transit vehicles as trains but these could also be buses, trams, or metros.) Let $S$ denote the set of transit stations. Let $t_{i j}^{p}$ be the (shortest) travel time by train between station $i \in S$ and $j \in S$, where the travel between two stations in the network can involve one train or multiple trains with transfers. In case the shortest route involves transfers then transfer and waiting times are included in the travel time. Note that for a cyclic timetable with identical

trains the travel time $t_{i j}^{p}$ is not dependent on the departure time from station $i$. We denote by $\theta_{s}^{-}(t)$ the last train departure at station $s \in S$ at or before time $t$ and by $\theta_{s}^{+}(t)$ the first train departure at station $s \in S$ at or after time $t$.

For presentational convenience, we only consider trips into the city center, i.e., the morning commute. It is straightforward to extend the concepts and technology to accommodate more extensive travel patterns. Consequently, we assume for each station $s \in S$ an access time, $\tau_{s}^{a}$, representing the time needed to walk from the drop-off location to the platform. Furthermore, we assume that a subset of the transit stations, $S_{P} \subseteq S$, have a park-and-ride facility and for each station $s \in S_{P}$ a park time, $\tau_{s}^{p}$, representing the time needed to park and walk from the parking facility to the platform. Finally, we assume that when a rider (or 
a driver) uses public transport, he will always travel to the station that is closest to his final destination. Let $s_{r}$ be the closest station to the destination of rider $r$. Hence, using public transit is only feasible for rider $r$ if $s_{r}$ is within the maximum acceptable walking distance of his destination, i.e., when $d_{s_{r}} d_{r} \leq m_{r}$. For assessing the feasibility of matches involving public transport, it is convenient to calculate the latest time, $l_{r}^{s}$, at which a rider $r$ can arrive at the platform at station $s \in S$ and reach his destination at or before $l_{r}$, i.e., $l_{r}^{s}=\theta_{s}^{-}\left(l_{r}-\hat{t}_{s_{r}} d_{r}-t_{s s_{r}}^{p}\right)$.

We allow matches in which a driver picks up two riders for drop-off at a transit station (i.e., from two different pickup locations). However, to minimize the inconvenience for the driver, the two riders will be dropped off at the same transit station.

Thus, the transit service provider offers these types of matches:

- A ride-share match: a match between a rider and a driver in which the driver transports the rider from his origin to his destination;

- A transit match: a match between a rider and a driver in which the driver transports the rider to a transit station. Subsequently, the driver drives to his destination while the rider takes public transit to reach his final destination.

- A park-and-ride match: a transit match in which the driver parks his car and then uses public transport to reach his final destination

A ride-share match always involves one rider, but both a transit match and a park-and-ride match can involve one or two riders.

\subsection{Assessing the feasibility of a ride-share match}

A ride-share match involves a driver $i \in D$ and a rider $j \in R$. The departure time $e_{i}^{j}$ of a driver $i$ matched with rider $j$ depends on the announcement time of rider $j$, the earliest departure time of rider $j$, and the trip duration from $o_{i}$ to $o_{j}$ and is set as follows $e_{i}^{j}=\max \left(e_{i}, a_{j}, e_{j}-t_{o_{i} o_{j}}\right)$. This assures that driver $i$ does not wait for rider $j$ at $o_{j}$. The pickup time, $e_{j}^{i}$, of rider $j$ is $e_{i}^{j}+t_{o_{i} o_{j}}$ and the arrival time, $l_{j}^{i}$, at the rider's destination is $e_{j}^{i}+\tau+t_{o_{j} d_{j}}$. Driver $i$ will arrive at his destination at time $l_{i}^{j}=l_{j}^{i}+t_{d_{j} d_{i}}$. The ride-share match is feasible only if the trip time duration for driver $i$, i.e., $l_{i}^{j}-e_{i}^{j}$, is less than or equal to $T_{i}$. Also, driver $i$ and rider $j$ must arrive at their destinations before their latest arrival times $l_{i}$ and $l_{j}$, respectively. 


\subsection{Assessing the feasibility of a transit match}

A single-rider transit match involves a driver $i \in D$, a rider $j \in R$, and a transit station $s \in S$. The departure time of the driver, $e_{i}^{j}$, and the pickup time of the rider, $e_{j}^{i}$, are computed in the same way as for a rideshare match. The arrival time at station $s$ is $e_{j}^{i}+\tau+t_{o_{j} s}$. The driver arrives at his destination at time $l_{i}^{j}=e_{j}^{i}+t_{o_{j} s}+\tau+t_{s d_{i}}$. The rider arrives at his destination at time $l_{j}^{i}=\theta^{+}\left(e_{j}^{i}+\tau+t_{o_{j} s}+\tau_{s}^{a}\right)+t_{s s_{r}}^{p}+\hat{t}_{s_{r} d_{r}}$. The transit match is feasible if the rider arrives at the platform of the transit station in time, i.e., $e_{j}^{i}+\tau+t_{o_{j} s}+\tau_{s}^{a} \leq$ $l_{j}^{s}$, if the driver arrives at his destination in time, i.e., $l_{i}^{j} \leq l_{i}$, and the trip durations of the rider, i.e., $l_{j}^{i}-e_{j}^{i}$, and the driver, i.e., $l_{i}^{j}-e_{i}^{j}$, are less than or equal to $T_{j}$ and $T_{i}$, respectively.

A two-rider transit match involves a driver $i \in D$, riders $j, k \in R(j \neq k)$, and a transit station $s \in S$. Without loss of generality we assume that rider $j$ is picked up before rider $k$. To avoid waiting at $o_{j}$ and $o_{k}$, the driver departs at $e_{i}^{j k}=\max \left(e_{i}, a_{j}, e_{j}-t_{o_{i} o_{j}}, a_{k}, e_{k}-\left(t_{o_{i} o_{j}}+\tau+t_{o_{j} o_{k}}\right)\right)$. The pickup time $e_{j}^{i}$ of rider $j$ is $e_{i}^{j k}+t_{o_{i} o_{j}}$ and the pickup time $e_{i}^{k}$ of rider $k$ is $e_{j}^{i}+\tau+t_{o_{j} o_{k}}$. The arrival time at station $s$ is $e_{k}^{i}+\tau+t_{o_{k} s}$. The driver arrives at his destination at time $l_{i}^{j}=e_{k}^{i}+\tau+t_{o_{j} s}+t_{s d_{i}}$. The transit match is feasible only if both riders arrive at the platform at the transit station in time, if the driver arrives at his destination in time, and if the trip durations of the driver and the riders are less than the maximum acceptable trip durations.

Note that in the discussion above we have not explicitly considered the waiting time of a rider at the platform, e.g., $w_{j}^{i}=\theta^{+}\left(e_{j}^{i}+\tau+t_{o_{j} s}+\tau_{s}^{a}\right)-\left(e_{j}^{i}+\tau+t_{o_{j} s}+\tau_{s}^{a}\right)$ in the case of a single-rider transit match. However, it is easy to see that if the transit match is feasible, the waiting time at the transit station can be minimized by having the driver depart later. The delay in departure of the driver is bounded by $\min \left(w_{j}^{i}, l_{i}-\right.$ $\left.l_{j}^{i}, l_{j}^{s}-e_{j}^{i}-\left(\tau+t_{o_{j} s}+\tau_{s}^{a}\right)\right)$, i.e., the current waiting time, the remaining driver flexibility, and the remaining rider flexibility.

\subsection{Assessing the feasibility of a park-and-ride match}

Assessing the feasibility of a park-and-ride match is similar to assessing the feasibility of a transit match. We only have to account for the fact that after dropping off the rider(s) at the transit station, the driver continues using public transit rather than continues using his car. The first difference occurs in the calculation of the arrival time at the station platform: rather than using the access time $\tau_{s}^{a}$, we use the park time $\tau_{s}^{p}$ to account for the fact that the driver parks his car and then driver and rider(s) walk to the platform from there. The second difference is calculating the time that the driver arrives at his destination, which now involves public transit. 


\subsection{The matching problem}

As in Stiglic et al. [2015], we create a node for each driver $i \in D$ and each rider $j \in R$ and an edge connecting node $i$ and $j$ if there is a feasible match between driver $i$ and rider $j$. We also introduce nodes that represent pairs of riders $(j, k)$, where $j, k \in R$ and $j \neq k$. We add an edge connecting node $i$ and $(j, k)$ if there is a feasible match between driver $i$ and rider pair $(j, k)$. Each edge $e$ has two weights: number of riders in the match, $v_{e}$, and the additional driving distance for drivers, $\delta_{e}$ (note that this value may be negative in park-and-ride matches because the driver uses public transit to reach his destination from the park-and-ride station). Note that a transfer match between a driver and a rider (or a driver and a pair of riders) may not be unique, because there may exist a number of feasible transfer matches involving different stations and different departures. Similarly, a park-and-ride match between a driver and a rider (or a driver and a pair of riders) may not be unique. We only consider the match with the lowest driving distance for that combination of the driver and rider(s).

Let $E$ represent the set of all edges in the bipartite graph and let the binary decision variable $x_{e}$ for edge $e \in E$ indicate whether the edge is in an optimal matching $\left(x_{e}=1\right)$ or not $\left(x_{e}=0\right)$. Furthermore, let $E_{i}$ and $E_{j}$ represent the set of edges in $E$ associated with driver $i$ and rider $j$, respectively. Then, our ride-share matching problem with the objective of maximizing the number of matched riders can be formulated as the following integer program:

$$
\max z_{1}=\sum_{e \in E} v_{e} x_{e}
$$

subject to

$$
\begin{aligned}
& \sum_{e \in E_{i}} x_{e} \leq 1 \quad \forall i \in D, \\
& \sum_{e \in E_{j}} x_{e} \leq 1 \quad \forall j \in R, \\
& x_{e} \in\{0,1\} \quad \forall e \in E .
\end{aligned}
$$

Objective function (1) maximizes the number of matched riders. Constraints (2) and (3) assure that each driver and each rider is only included in at most one match in an optimal matching, respectively.

To obtain a matching that minimizes the total increase in driving distance for all drivers, the objective 
should be replaced by:

$$
\min z_{2}=\sum_{e \in E} \delta_{e} x_{e}
$$

Since both objectives, i.e., maximizing the number of matched riders and minimizing the total trip time increase for all participants, are relevant, we take both objectives into account in a hierarchical fashion, where we consider $z_{1}$ as the primary objective and $z_{2}$ as the secondary objective. We first solve (1) subject to (2) - (4). Let $z_{1}^{*}$ be the number of matched riders. We then solve (5) subject to (2) - (4) plus the additional constraint $\sum_{e \in E} v_{e} x_{e} \geq z_{1}^{*}$.

\section{Solution approach}

Our ride-matching algorithm consist of a match identification phase and an optimization phase in which the optimal matching is determined (based on the set of feasible matches). Match identification is performed separately for each match type, i.e. ride-share matches, transfer matches, and park-and-ride transfer matches. To identify feasible matches with two riders, we take advantage of the property that a match between driver $i$ and riders $j$ and $k, j \neq k$, can be time feasible (but not necessarily) only if the matches between driver $i$ and rider $j$ and driver $i$ and rider $k$ are both time feasible.

\subsection{Determining feasible ride-share matches}

Single ride-share match identification is a relatively simple task. In this paper, we perform a straightforward enumeration of all possible driver-rider pairs and identify those that are feasible by checking the conditions outlined in Section 3.1.

\subsection{Determining feasible transit and matches}

The identification of feasible transit matches starts with several preprocessing procedures. For each announcement $a \in A$, we determine the closest public transit station $s \in S$ to the destination location $d_{a}$. This is done by performing a query in a $k-d$ tree in which all transit station locations are stored. Let $s_{a}$ denote the closest public transit station to the destination location for announcement $a$. The set of rider announcements $R_{p}:=\left\{j \in R \mid d_{s_{j}} d_{j} \leq m_{j}\right\}$ is considered in the determination of potential transfer matches and the set of driver announcements $D_{p}:=\left\{i \in D \mid d_{s_{i} d_{i}} \leq m_{i}\right\}$ is considered in the determination of potential parkand-ride transfer matches. Note that all driver announcements $d \in D$ are considered in the determination of 
potential transfer matches.

The next preprocessing procedure serves to find for each rider $j \in R_{p}$ the set of feasible origin transit stations $S_{j}$ and the set of feasible departures at each feasible origin station $s \in S_{j}$. For a rider $j \in R_{p}$, a train departure time $t$ at origin station $s \in S_{j}$ is feasible if $e_{j}+\tau+t_{o_{j} s} \leq t$ and $t \leq l_{j}-\hat{t}_{s_{j} d_{j}}-t_{s s_{j}}^{p}$, i.e., the earliest possible arrival time at the station is less than $t$ and the earliest arrival at the destination departing at or after $t$ is less than $l_{j}$. All feasible departures are stored for each individual rider separately.

In another preprocessing step, we find for each driver $i \in D$ the set of feasible transit stations $S_{d}$ and the set of feasible departures at each feasible transit station $s \in S_{d}$ that he can visit. For a driver $i \in D$, a transit station $s$ is feasible if he can visit this station on his way from his origin to his destination location without exceeding his maximum acceptable trip duration. A station departure $t$ is feasible if the arrival of the driver occurs at or before $t-\tau_{s}^{a}$. To identify such departures, we build an implied time window $\left[e_{s}^{i}, l_{s}^{i}\right]$ at station $s$ with earliest arrival time $e_{s}^{i}=e_{i}+t_{o_{i} s}+\tau$ and the latest departure time $l_{s}^{i}=l_{i}-t_{s d_{i}}$. All departures $t$ for which $t-\tau_{s}^{a} \in\left[e_{s}^{i}, l_{s}^{i}\right]$ are feasible. A lookup table is constructed that enables one to query potentially feasible drivers based on origin station and departure. Feasible single rider transit matches are identified for each rider by inspecting all feasible stations and feasible train departures for that rider and retrieving all potentially compatible drivers. The feasibility of a particular match is checked by examining the conditions that are outlined in Section 3.2.

\subsection{Determining feasible park-and-ride matches}

To identify feasible park-and-ride matches, a slightly different preprocessing procedure for the drivers is performed in comparison to the one described in the previous section. The (only) difference is that the latest departure time $l_{s}^{i}$ is calculated using transit trip duration from $s$ to $s_{i}$, the destination station of the driver, i.e., $l_{s}^{i}=l_{i}-\hat{s}_{i} d_{i}-t_{s s_{i}}^{p}-\tau_{s}^{p}$. Based on the preprocessed feasible departures of the riders and drivers, feasible single-rider park-and-ride matches are identified for each rider by inspecting all feasible stations and feasible train departures for that rider and retrieving all potentially compatible drivers. The feasibility of a particular park-and-ride match is checked by examining all conditions that are outlined in Section 3.3.

\subsection{Determining feasible transit and park-and-ride matches with two riders}

Once all feasible single-rider transit (or park-and-ride) matches are known, we group all matches of this type using a hash map on driver - station combinations. We examine each individual driver - station combination and construct all potential pairs of riders. We evaluate all possible combinations of departures for a driver - station - rider pair combination to identify and record the best feasible one according to total trip time 
increase for the participants in a match. Once all feasible transit and park-and-ride matches are known, we determine the best match for a particular driver - rider pair match.

\section{A computational study}

In this section, we report the results of our computational study to assess the possible benefits and synergies that can arise as a result of integrating a ride-sharing system and a public transit system.

\subsection{Generation of instances}

We focus on a rectangular metropolitan area of 20 by 10 miles that features a circular urban center with a radius of 2.5 miles and a sprawling suburban area. We consider a stylized transit network that captures the key features of a real-world commuter train network, such as the Bay Area Rapid Transit (BART) network in the San Francisco region. The radial route network consists of two commuter train lines and four urban rapid transit lines. Transfers between the different train lines are only possible at the transfer hub, which is located in the center of the urban area. The inter-station distance is approximately $2.25 \mathrm{mi}$ on commuter lines and $0.75 \mathrm{mi}$ on the urban transit lines. Figure 2 illustrates the transit lines, stations, and the urban and suburban regions. The figure also indicates the eight park-and-ride stations.

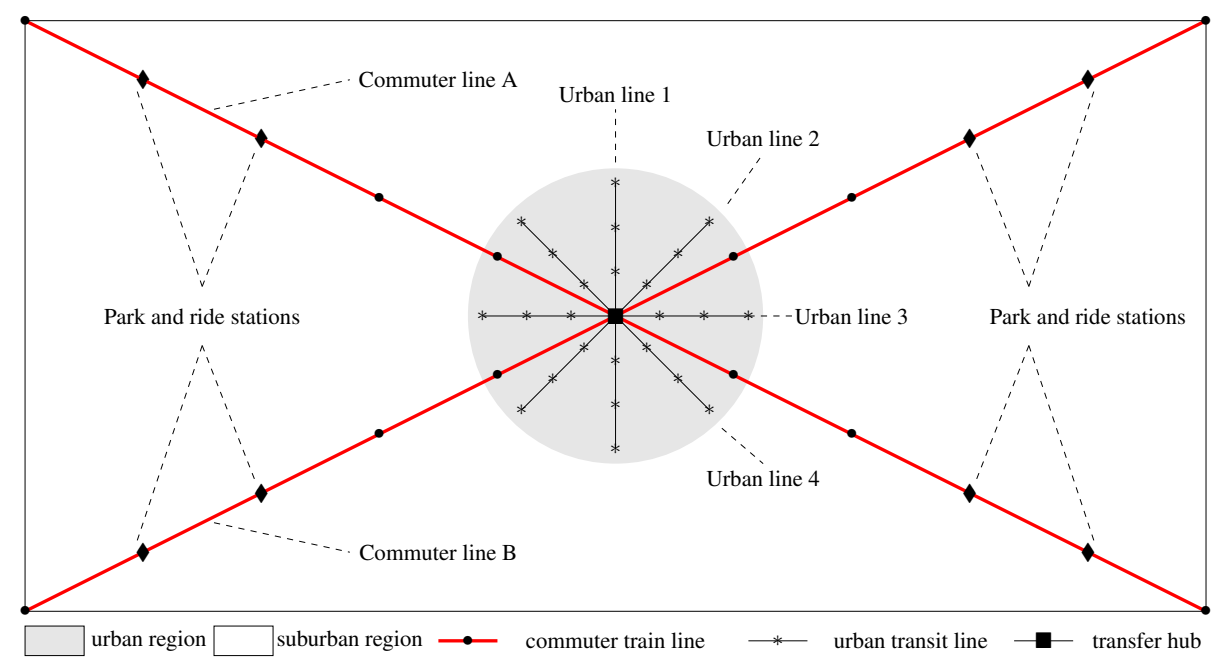

Figure 2: Representation of public transit network and urban/suburban regions.

We generate $n$ participant trips in which the probability of a driver or rider trip is equally likely. For each trip announcement, we generate an earliest departure time based on a truncated normal distribution with a standard deviation of 30 minutes. To create commuter trips, we randomly draw origins from the complete region and draw destinations from the urban center. Since we assume that it is unlikely that participants with 
very short trips will participate, we use rejection sampling to filter out the trips that are shorter than 1 mile.

Travel times on roads are calculated using Euclidean distances with a 30\% uplift. We assume an average driving speed of $20 \mathrm{mph}$, which represents the travel speed in a congested urban area. We consider a pickup time of two minutes for each rider that is pickup by a driver. We assume a walking speed of 4 feet per second (LaPlante and Kaeser, 2004). The maximum walking distance for the rider to or from a transit station is 0.5 miles, which corresponds to 11 minutes of walking at this speed.

We assume a cyclic schedule with the same departure frequency for all transit lines, with a default departure frequency of 15 minutes. The line schedules are synchronized at the hub location so that all transfers between lines take the same amount of time. The average speed of the commuter train is $40 \mathrm{mph}$ and the average speed of the urban transit train is $20 \mathrm{mph}$. All trains have a dwell time of one minute for regular stations and three minutes at the hub station to allow for transfers. We assume that it takes two minutes to enter and leave a transit station which captures walking to and from the platform, with an additional two minute service time for all park-and-ride matches to account for the time that is needed to park the vehicle. The characteristics of the base case instances are summarized in Table 1.

Table 1: Characteristics of the base case instances.

\begin{tabular}{lr}
\hline Trip pattern: & suburb to center \\
Avg. number of participants: & 1000 \\
Driver-rider ratio: & 0.5 \\
Matching flexibility: & $20 \mathrm{~min}$ \\
Scheduling flexibility: & $15 \mathrm{~min}$ \\
Driver detour flexibility: & $25 \%$ \\
Rider flexibility: & $50 \%$ \\
Maximum number of driver stops: & 3 \\
Avg. driver trip distance: & $8.0 \mathrm{mi}$ \\
Avg. driver trip duration: & $24.1 \mathrm{mi}$ \\
Max. walk distance to transit station: & $0.5 \mathrm{mi}$ \\
Walk speed: & $4 \mathrm{ft} / \mathrm{s}$ \\
Car speed: & $20 \mathrm{mi} / \mathrm{h}$ \\
Suburban train speed: & $40 \mathrm{mi} / \mathrm{h}$ \\
Urban train speed: & $20 \mathrm{mi} / \mathrm{h}$ \\
Vehicle capacity: & $2 \mathrm{seats}$ \\
Pickup time per rider: & $2 \mathrm{~min}$ \\
Transfer time street to platform: & $2 \mathrm{~min}$ \\
Additional transfer time for park-and-ride: & $2 \mathrm{~min}$ \\
Train dwell time: & $1 \mathrm{~min}$ \\
Train hub dwell time: & $3 \mathrm{~min}$ \\
Frequency of train departures: & $15 \mathrm{~min}$ \\
Number of stations: & 41 \\
Number of park-and-ride stations: & 8 \\
\hline
\end{tabular}




\subsection{Computational results}

The main aim of this research is to analyze and quantify the benefits of integrating ride-sharing with public transit and to determine what value this can create for different stakeholders, e.g., public transit agencies, ride-share providers, and system participants. We use the optimization results to compute and evaluate a number of metrics to gain insight into the system performance and the potential of ride-sharing to enhance mobility and to increase the use of public transport. In all experiments, we either use the base case setting or a setting in which one of the characteristics is varied in order to assess the sensitivity of the system performance to this characteristic.

The algorithm for generating feasible matches is implemented in $\mathrm{C}$ and the simulation framework is implemented in Python 3.4. CPLEX 12.6 is used for solving matching problems. All base case instances are solved within a few seconds, with solution times of up to a minute for the largest instances with 2000 participants.

\subsection{Benefits of an integrated system}

To evaluate the benefits of integrating ride-sharing and public transit, we analyze the matching rates for a number of different settings. As a benchmark, we consider a setting in which only door-to-door rideshare matches are generated, denoted by RS. Next, we consider settings in which both ride-share and transit matches are generated, where TRS1 denotes the setting in which only single-rider transit matches are generated and TRS2 denotes the setting in which transit matches with one or two riders are generated. Finally, PTRS denotes the setting in which, in addition to the matches considered in TRS2, a driver may opt to take public transport after dropping off riders at a transit station, i.e., park-and-ride matches. The results for the base case can be found in Table 2, where we report averages over 10 randomly generated instances. When we report the percentage of transit and $\mathrm{P}+\mathrm{R}$ matches, it is relative to the number of matches, i.e., if 80 out of a 100 riders are matched and 36 of them are dropped off at a transit station by drivers who then drive to their final destinations, and 4 of them were dropped off at a transit station by drivers who then take public transport to their destinations, the matching rate is $80 \%$, the transit matching rate is $45 \%$, and the $\mathrm{P}+\mathrm{R}$ matching rate is $5 \%$. Furthermore, when we report travel time increases for riders, it is relative to the travel time they would have experienced if they had driven themselves.

We observe that integrating ride-sharing and public transit can have significant benefits. The average number of matched riders (our primary objective) increases from $66.8 \%$ to $83.8 \%$. Interestingly, the average length of the driver detour (our secondary objective) in an integrated system is smaller than in a ride-share 
Table 2: Results for different ride-share settings (avg. over 10 random base case instances).

\begin{tabular}{l|cccc}
\hline & RS & TRS1 & TRS2 & PTRS \\
\hline Riders & & & & \\
Matching rate (\%): & 66.8 & 74.0 & 83.7 & 83.8 \\
Transit matches (\%): & 0 & 32.4 & 37.2 & 33.9 \\
P+R matches (\%): & 0 & 0 & 0 & 4.0 \\
$\Delta$ travel time (\%): & 0 & 7.3 & 7.4 & 7.5 \\
\hline Drivers & & & & \\
Matching rate (\%): & 68.3 & 75.5 & 73.8 & 74.2 \\
Transit matches (\%): & 0 & 32.4 & 27.3 & 25.1 \\
P+R matches (\%): & 0 & 0 & 0 & 3.3 \\
$\Delta$ travel time (\%): & 19.1 & 17.1 & 21.6 & 21.9 \\
$\Delta$ distance (\%): & 8.4 & 7.0 & 10.4 & 7.2 \\
\hline
\end{tabular}

only system, i.e., $7.2 \%$ compared to $8.4 \%$. Thus, not only is mobility enhanced, but the negative externalities associated with car travel, such as emissions and congestion, are reduced. This reduction in the average length of the driver detour is due, in part, to drivers deciding to use public transport. Maybe as important is the fact that the average increase in travel time for riders is relatively small, i.e., $7.5 \%$. This may encourage more people to consider public transport as a viable alternative to traveling by car.

Another important observation is that to reach a high matching rate, it is critical that a driver is willing to pick up and drop-off more than one rider (in our setting at most two and dropped off at the same transit station). If drivers are only willing to pick up and drop off a single rider, the matching rate is $74 \%$ whereas if drivers are willing to pick up and drop off two riders the matching rate is $83.7 \%$. Of course, this increase in matching rate comes at the expense of an increase in the length of the average driver detour (from $7.0 \%$ to $10.4 \%)$.

In Figure 3, we provide more details on the matches found for riders by showing the number of riders in the different types of matches for the four settings. We see, again, that transit matches involving two riders are critical to achieving high matching rates in the settings that allow them (TRS2 and PTRS). We see too that the number of riders in park-and-ride matches is small (only 17 on average).

The fact that allowing park-and-ride matches results in an increase in matching rate may, at first, seem surprising as it mostly impacts drivers. However, some transit matches are time feasible only when the driver use public transport, because the driver can reach his destination faster using public transport than by car. Another factor contributing to the small number of drivers choosing to use public transport is the use of hierarchical optimization, i.e., maximizing the matching rate followed by minimizing (total) driver distance subject to the constraint that the matching rate cannot decrease. The constraint that the matching rate cannot decrease is restrictive and leaves little flexibility for reducing the detour distance. If we would be willing to 


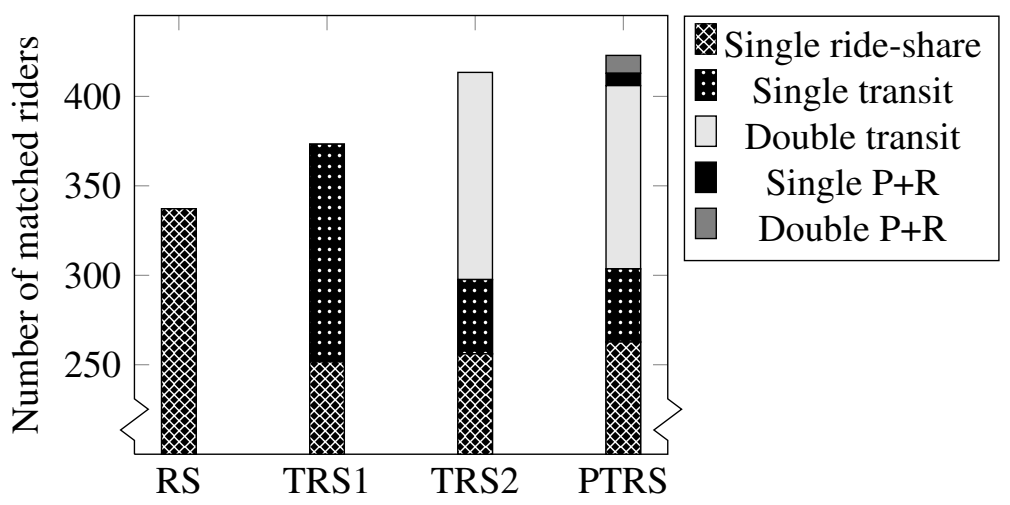

Figure 3: Number of matched riders in different match types.

accept slightly smaller matching rates, it is possible that we would see many more drivers opting for public transport. Finally, the location of the transit stations offering park-and-ride, which in our transit network are relatively far from the urban center, (negatively) impacts the benefits of the park-and-ride option.

To provide further insight into the opportunities offered by an integrated system, we look at the trips associated with the transfer and park-and-ride matches generated for a single instance. Figure 4 shows the paths from the riders' origins to their transit stations. For matches that involve two riders, the trip from the origin of the first rider to the origin of the second rider is represented by a dotted line and the trip from the second rider's origin to the transit station is represented by a solid line. Interestingly, we see that only about $25 \%$ of the riders is dropped off at the station that is closest to their origin. It is often more convenient for a driver to use a transit station closer to the urban center. As a consequence, the public transit trips for riders tend to be relatively short. This also suggests, that park-and-ride facilities at transit stations close to the urban center may significantly increase use of public transport by drivers.

To further analyze these trips, we break-down, in Figure 5, the single-rider transit matches into: (1) waiting time at home (from the earliest possible departure time), (2) transfer time to station, (3) waiting time at station, (4) transit trip duration, and (5) walking time from destination transit station to the destination. Note that the waiting time at a transit station includes the time to reach the platform. Similarly, the walking time from a transit station to the final destination includes the time to leave the platform. The waiting time at home is presented for completeness sake; it is, of course, not part of the rider's actual trip. We see that the average travel time of these riders on the transit lines is relatively small, i.e., 12-13 minutes, which is approximately $30 \%$ of the average total trip duration.

In the next subsections, we evaluate the impact of varying instance characteristics. We will report results only for the most and the least restricted ride-share settings, i.e., RS and PTRS. 


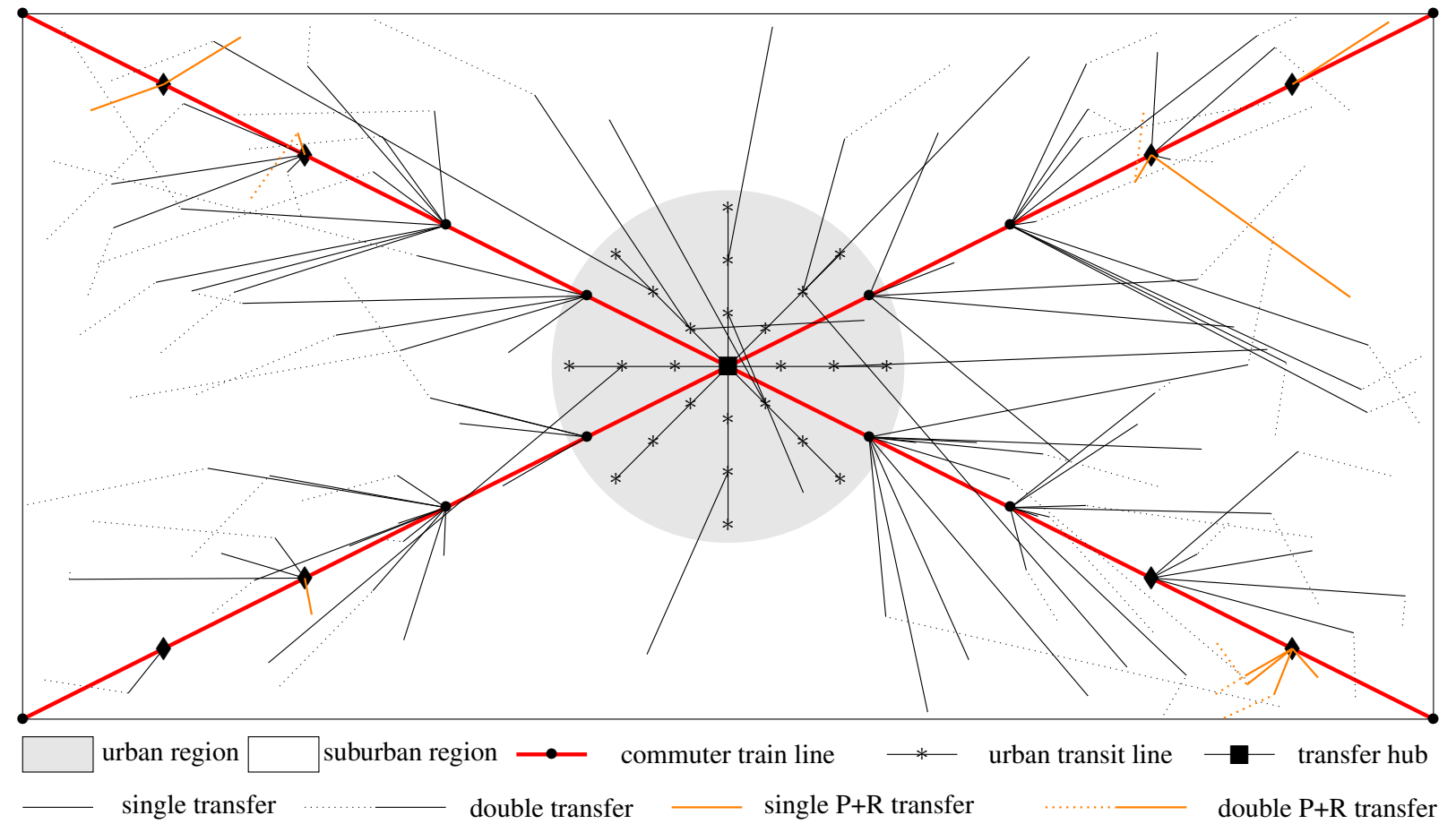

Figure 4: Map of rider paths to transit stations for one of the base case instances for the PTRS setting.

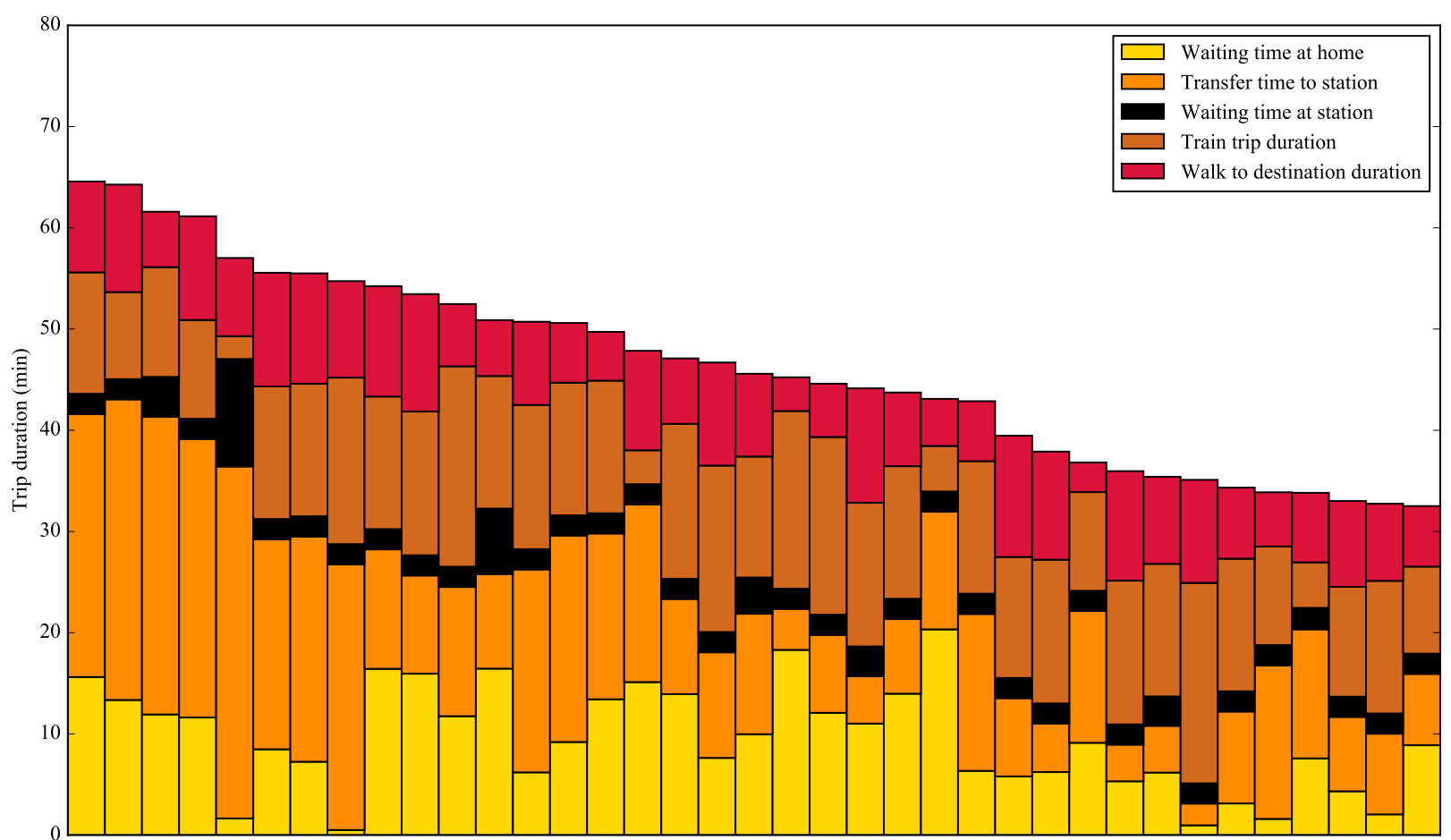

Figure 5: Breakdown of trip durations and waiting at home for all riders matched in single transit matches in the optimal solution for one of the base case instances. 


\subsection{Impact of driver matching flexibility}

In this section, we evaluate the impact of the matching flexibility of drivers, i.e., their willingness to extend the latest arrival time at their destination. Table 3 shows, unsurprisingly, that a lower driver matching flexibility (i.e., 10 mins.) limits the value of integrating ride-sharing with public transit, because fewer tworider transit matches are feasible (there is simply not enough time to accommodate more than one rider).

Table 3: Results for different driver matching flexibilities (Avg. over 10 random instances).

\begin{tabular}{l|cc|cc|cc}
\hline & \multicolumn{2}{|c|}{10 min } & \multicolumn{2}{c|}{ 15 min } & \multicolumn{2}{c}{ 20 min } \\
\hline & RS & PTRS & RS & PTRS & RS & PTRS \\
\hline Riders & & & & & & \\
Matching rate (\%): & 64.9 & 78.8 & 66.8 & 83.8 & 66.3 & 84.0 \\
Transit matches (\%): & 0 & 34.3 & 0 & 33.9 & 0 & 38.7 \\
Drivers & & & & & & \\
Matching rate (\%): & 65 & 70.8 & 68.3 & 74.2 & 66.7 & 73.7 \\
P+R matches (\%): & 0 & 3.0 & 0 & 3.3 & 0 & 3.8 \\
$\Delta$ distance (\%): & 8.1 & 6.4 & 8.4 & 7.2 & 8.5 & 7.0 \\
\hline
\end{tabular}

\subsection{Impact of density}

In this section, we investigate how the number of system participants affects the performance. Table 4 shows, as expected, that the matching rate increases significantly when the number of system participants increases; the net improvements in rider matching rate are 14.4 to 17.6 percentage points.

Table 4: Results for different numbers of participants in the system (Averaged over 10 random instances).

\begin{tabular}{l|cc|cc|cc}
\hline & \multicolumn{2}{|c|}{$\mathbf{5 0 0}$} & \multicolumn{2}{c|}{$\mathbf{1 0 0 0}$} & \multicolumn{2}{c}{ 2000 } \\
\hline & RS & PTRS & RS & PTRS & RS & PTRS \\
\hline Riders & & & & & & \\
Matching rate (\%): & 53.5 & 67.9 & 66.8 & 83.8 & 76.0 & 93.7 \\
Transit matches (\%): & 0 & 39.0 & 0 & 33.9 & 0 & 36.5 \\
Drivers & & & & & & \\
Matching rate (\%): & 56.3 & 63.6 & 68.3 & 74.2 & 74.1 & 78.7 \\
P+R matches (\%): & 0 & 4.6 & 0 & 3.3 & 0 & 4.3 \\
$\Delta$ distance (\%): & 7.1 & 4.7 & 8.4 & 7.2 & 8.8 & 6.7 \\
\hline
\end{tabular}

\subsection{Impact of transit system parameters}

In this section, we study the impact of the characteristics of the transit line on the performance of the system. We consider three speed scenarios for the suburban trains: $30 \mathrm{mph}, 40 \mathrm{mph}$ (base case) and $50 \mathrm{mph}$ and three 
departure frequencies for all transit lines: every 5, 15 (base case) or 25 minutes. Figure 6 shows the rider matching rates for the nine combinations. As expected, we see that more frequent departures and faster trains improve the performance of the system and create more rider matches. The results also suggest that the departure frequency is more important than speed, i.e. there is only a marginal benefit from increasing the suburban train speed from $40 \mathrm{mph}$ to $50 \mathrm{mph}$.

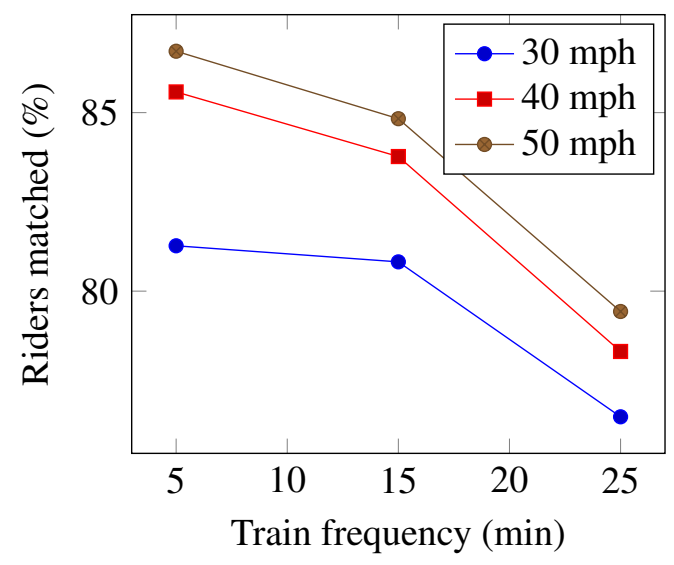

Figure 6: Rider match rates for different transit line characteristics

\section{Final remarks}

Our study has shown that the integration of a ride-sharing system and a public transit system can significantly enhance mobility, increase the use of public transport, and reduce the negative externalities associated with car travel.

We have found that driver willingness to pick up and drop off more than one rider is critical to the system performance. We have investigated only the simplest and most convenient (from a driver perspective) variant, i.e., driver willingness to pick up two riders and drop them off at the same transit station. Further investigation of more flexible variants is warranted. We have also observed the potential of park-and-ride facilities, but more experimentation is needed to fully understand the benefits, i.e., the location and number of transit stations with park-and-ride facilities as well as the use of different objective hierarchies when generating ride-share and transit matches.

Other opportunities for further research include analyzing more complex transit systems, the use of meeting points in order to streamline the process of picking up riders, and the integration of (free) bikesharing systems. 


\section{References}

N. A. H. Agatz, A. Erera, M. P. W. Savelsbergh, and X. Wang. Dynamic ride-sharing: A simulation study in metro atlanta. Transportation Research Part B, 45(9):1450-1464, 2011.

N. A. H. Agatz, A. Erera, M. P. W. Savelsbergh, and X. Wang. Optimization for dynamic ride-sharing: A review. European Journal of Operational Research, 223(2):295-303, 2012.

R. Cayford and Y Yim, Y.B. Personalized demand-responsive transit service. Technical report, University of California, Berkley, April 2004.

Brian J Coltin and Manuela Veloso. Towards ridesharing with passenger transfers. In Proceedings of the 2013 international conference on Autonomous agents and multi-agent systems, pages 1299-1300. International Foundation for Autonomous Agents and Multiagent Systems, 2013.

Florian Drews and Dennis Luxen. Multi-hop ride sharing. In Proceedings of the Sixth Annual Symposium on Combinatorial Search, pages 71-79. AAAI Press, 2013.

European Environment Agency. Occupancy rates of passenger vehicles. Technical report, European Environment Agency, 2010.

M. Furuhata, M. Dessouky, F. Ordonez, M.E. Brunet, X. Wang, and S. Koenig. Ridesharing: The stateof-the-art and future directions. Transportation Research Part B: Methodological, 57(0):28 - 46, 2013. ISSN 0191-2615.

Wesam Herbawi and Michael Weber. Evolutionary multiobjective route planning in dynamic multi-hop ridesharing. In Evolutionary Computation in Combinatorial Optimization, pages 84-95. Springer, 2011.

D. Koffman. Operational experiences with flexible transit services. Technical report, Transportation Research Board, January 2004.

A. Lee and M. Savelsbergh. An extended demand responsive connector. EURO Journal on Transportation and Logistics, pages 1-26, 2014.

Alan Lee and Martin Savelsbergh. Dynamic ridesharing: Is there a role for dedicated drivers? Transportation Research Part B: Methodological, 2015. doi: http://dx.doi.org/10.1016/j.trb.2015.02.013.

X. Li and L. Quadrifoglio. Feeder transit services: Choosing between fixed and demand responsive policy. Transportation Research Part C: Emerging Technologies, 18(5):770 - 780, 2010. 
X. Li and L. Quadrifoglio. 2-vehicle zone optimal design for feeder transit services. Public Transport, 3(1): 89-104, 2011.

B.S. McKenzie. Public transportation usage among u.s. workers: 2008 and 2009. Technical report, U.S. Census Bureau, October 2010.

Colin Murphy. Shared mobility and the transformation of public transit. Technical report, 2016.

G. Murray, M.E. Chase, E. Kim, and M. McBrayer. Ridesharing as a complement to transit: A synthesis of transit practice. Technical report, Transportation Research Board, February 2012.

S.E. Polzin and A.E. Pisarski. Commuting in america 2013. Technical report, American Association of State Highway and Transportation Officials, April 2013.

L. Quadrifoglio and L. Xiugang. A methodology to derive the critical demand density for designing and operating feeder transit services. Transportation Research Part B: Methodological, 43(10):922 - 935, 2009.

M. Stiglic, N. Agatz, M. Savelsbergh, and M. Gradisar. The benefits of meeting points in ride-sharing systems. Transportation Research Part B: Methodological, 82:36 - 53, 2015.

M. Stiglic, N. Agatz, M. Savelsbergh, and M. Gradisar. Making dynamic ride-sharing work: The impact of driver and rider flexibility. Transportation Research Part E: Logistics and Transportation Review, 91:190 $-207,2016$.

Xiaoqing Wang, Maged Dessouky, and Fernando Ordonez. A pickup and delivery problem for ridesharing considering congestion. Transportation Letters: The International Journal of Transportation Research, pages 1942787515Y-0000000023, 2015. 